\title{
High-energy $\gamma$-rays from the Milky Way: 3D Interstellar Emission Models with GALPROP
}

\section{Troy A. Porter*}

W. W. Hansen Experimental Physics Laboratory and Kavli Institute for Particle Astrophysics and Cosmology, Stanford University, Stanford, CA 94305, USA

E-mail: tporterestanford.edu

\section{Guollaugur Jóhannesson}

Science Institute, University of Iceland, IS-107 Reykjavik, Iceland and AlbaNova Univ. Center Nordita, Roslagstullsbacken 23, SE-106 91 Stockholm, Sweden

E-mail: gudlaugu@hi.is

\section{Igor V. Moskalenko}

W. W. Hansen Experimental Physics Laboratory and Kavli Institute for Particle Astrophysics and Cosmology, Stanford University, Stanford, CA 94305, USA

E-mail: imosestanford.edu

\begin{abstract}
High-energy $\gamma$-rays of interstellar origin are produced by the interaction of cosmic-ray (CR) particles with the diffuse gas and radiation fields in the Galaxy. The main features of this emission are well understood and are reproduced by existing CR propagation models employing a 2D galactocentric cylindrically symmetric geometry. However, the high-quality data from instruments like the Fermi Large Area Telescope reveal significant deviations from the 2D model predictions on few to tens of degree scales, indicating that the details of the Galactic spiral structure should be included and thus require 3D spatial modelling. In this contribution the high-energy interstellar emissions from the Galaxy are calculated using the latest release of the GALPROP code for the first time employing full 3D spatial models for the CR source, interstellar gas, and interstellar radiation field (ISRF) densities. The interstellar emission models that include arms and bulges for the CR source and ISRF densities provide plausible physical interpretations for features found in the residual maps from high-energy $\gamma$-ray data analysis. The 3D models provide a more realistic basis for interpreting the non-thermal interstellar emissions toward the inner Galaxy and about the Galactic centre.
\end{abstract}

7th Fermi Symposium 2017

15-20 October 2017

Garmisch-Partenkirchen, Germany

\footnotetext{
*Speaker.
} 


\section{Introduction}

The high-energy $\gamma$-ray sky is dominated by the emissions produced by cosmic-ray (CR) particles interacting with matter and radiation fields in the interstellar medium (ISM) of the Milky Way. Observations of these interstellar emissions began with the OSO-III satellite in the late 1960s, and were followed by the space-borne experiments SAS-2 and COS-B in the early- and mid-1970s, COMPTEL and EGRET on the Compton Gamma-Ray Observatory (CGRO) (1990s), and the present-day Fermi-LAT. Each of these instruments has represented a significant advance over its predecessor, with the Fermi-LAT providing the highest-sensitivity data so far for $\gtrsim 30 \mathrm{MeV}$ energies.

Physical modelling codes like GALPROP $[1,2,3,4]$ can reproduce the general features of the interstellar $\gamma$-ray emission over the whole sky, showing that the CR physics and interactions producing it are well understood. However, it is the residuals from when interstellar emission models (IEMs) are subtracted from the data that provide the potential for identifying new phenomena in high-energy $\gamma$-rays. Their understanding requires a careful assessment of the the modelling inputs, in particular those related to the CR source and ISM densities.

To date the most extensive study of high-energy IEMs has been made by the Fermi-LAT team [5] using a grid of 128 a-priori GALPROP models normalised to reproduce local CR data. The grid entries are categorised by $4 \mathrm{CR}$ source spatial density models from the literature (supernova remnants, 2 pulsar, and OB-stars), multiple CR propagation halo sizes, and other parameters related to the interstellar gas. These IEMs employ a 2D galactocentric cylindrically symmetric spatial geometry, which has been the norm since the CGRO-era due to the limited quality of the $\gamma$-ray data, information on ISM distributions, and computing resources available. Examination of the residual maps developed in that work shows $\sim$ few to tens of degrees scale features that are asymmetric about the meridian defined by Galactic longitude $l=0^{\circ}$ and the Galactic plane. Some of them are likely related to large-scale structure in the CR and ISM distributions that are not properly accounted for by the 2D-based IEMs.

The GALPROP code has been capable of 3D CR propagation calculations since the beginning $[2,6,7]$ but this mode has had limited usage because of the available data quality and computing resources necessary. Even the current data and theory does not provide for a complete 3D model of the ISM to be built. Thus, studying the effects of 3D structures on various observables is still at its early stages.

In this contribution, a study is made of the high-energy interstellar emissions calculated using 3D models for the CR source and ISM densities in the Galaxy. Three CR source density models are considered that have the injected CR power distributed as an axisymmetric smooth disc, 50/50\% smooth disc/spiral arms, or $100 \%$ spiral arms. A new 3D spatial density distribution for the interstellar gas is developed based on optimising model parameters using a maximum-likelihood fit to the H I LAB survey [8] and the DHT CO survey [9]. Two 3D ISRF models termed R12 and F98 that have been recently developed [10] are also used. The new release of the GALPROP code (v56) is employed using these CR and ISM density models as inputs for the CR propagation and high-energy interstellar emission calculations. With the addition of a population of CR sources distributed according to the stellar bulge/bars of either of the R12 and F98 ISRF models, the models with spiral arms provide a plausible physical explanation for the puzzling results from the analysis 
of high-energy $\gamma$-ray toward the inner Galaxy obtained by the Fermi-LAT team in [11].

\section{Calculations and Results}

The focus in this work is on the CR induced $\gamma$-ray emission from different realisations of the CR source and ISM distributions. The propagation model is therefore limited to diffusive re-acceleration with an isotropic and homogeneous diffusion coefficient that has a power-law dependence with rigidity. The CR injection spectra are modelled as rigidity-dependent broken power laws ${ }^{1}$ with parameters derived as by a recent study [12]. Electrons, protons, and He each have two breaks while elements with $Z>2$ are modelled with a single break. The extra break for the lowmass elements is to model the spectral change observed at rigidities above $100 \mathrm{GV}[13,14]$. The CR source density model is $100 \%$ disc (2D), $50 \% / 50 \%$ disc/spiral arm, or $100 \%$ spiral arm, where the disc and arms have the same exponential scale-height (200 pc) perpendicular to the Galactic plane. The smooth disc spatial density follows the radial distribution of pulsars as given by [15]. The spiral arm spatial density follows that of the 4 major arms in the R12 model [10] and assumes an identical injection of CR power by each arm. The CR source density models are termed SA0, SA50, and SA100, following the proportion of injected CR luminosity by the spiral arms. The normalisation for the injected CR power in each is obtained by requiring the propagated CR intensities agree with the local CR observations ${ }^{2}$, where all calculations use the IAU recommended Sun-GC distance of $R_{S}=8.5 \mathrm{kpc}[16]$.

For each of the SA0, SA50, and SA100 CR source densities the propagation model parameter tuning is made using a maximum-likelihood fit employing data from AMS-02, HEAO3-C2, Voyager-1, and PAMELA assuming a fixed halo size $z_{h}=6 \mathrm{kpc}$, where full details of the method are given in [10]. Because of the known covariance between the CR source and gas density models when normalising to the $\mathrm{CR}$ data [5] the propagation model parameter tuning is done separately for each CR source and gas density model (2D/3D). Following this methodology the resulting CR spectra for all source density models are within $\sim 5 \%$ of each other, and agree well with the data and are generally well within the data uncertainties, thus ensuring an 'apples-to-apples' comparison for the high-energy interstellar emissions predictions.

High-energy interstellar emissions are calculated using GALPROP for the SA0, SA50, and SA100 source density models, the standard 2D [5] and R12/F98 3D ISRF models, and the standard 2D and newly-derived 3D gas density models. The $\gamma$-rays are calculated from $1 \mathrm{MeV}$ to $100 \mathrm{GeV}$ energies using a logarithmic energy grid with 10 bins/decade spacing. Higher $\gamma$-ray energies correspond to $\mathrm{CRs}$ with energies $\gtrsim 1 \mathrm{TeV}$ where the steady-state source injection paradigm employed in this paper is less valid [7]. All calculations of the IC contribution use the anisotropic scattering cross section [17] that accounts for the full directional intensity distribution for each of the Std, R12, and F98 ISRF models. The SA0 CR source density and standard 2D gas and ISRF density models (SA0-Std/2Dgas) is used as the reference case. This combination corresponds to the 2D CR source and ISM density scenario that has been the standard approach for interstellar emission modelling in the past.

\footnotetext{
${ }^{1}$ Parameterisation: $q(R) \propto R^{\gamma_{0}}$ for $R<R_{1}, q(R) \propto R^{\gamma_{1}}$ for $R_{1}<R<R_{2}$, and $q(r) \propto R^{\gamma_{2}}$ for $R>R_{2}$.

${ }^{2}$ The proton and $\mathrm{e}^{-}$flux is normalised at the Solar location at a kinetic energy of $100 \mathrm{GeV}$.
} 

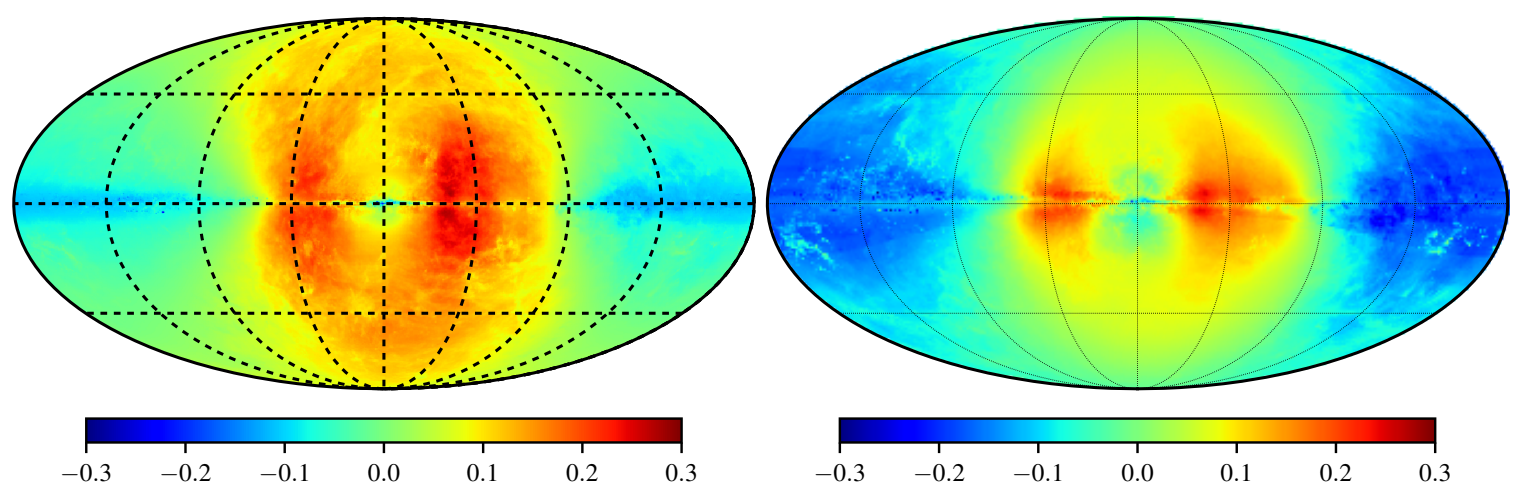

Figure 1: Fractional residual map at $1.2 \mathrm{GeV}$ for the SA100-R12/2Dgas (left) and SA100-Std/3Dgas (right) CR source and ISM density models with respect to the SA0-Std/2Dgas reference case. The map is in Galactic coordinates with $l, b=0^{\circ}, 0^{\circ}$ at the centre. The longitude meridians and latitude parallels have $45^{\circ}$ spacing.

Figure 1 shows the fractional residual map obtained for the SA100-R12/2Dgas (left) and SA100-Std/3Dgas (CR-ISRF/gas) model combinations, respectively, for a $\gamma$-ray energy of $1.2 \mathrm{GeV}$. For the 3D CR/ISRF scenario (left), toward the inner Galaxy a doughnut-like feature with excess emission concentrated near $l \sim \pm 45^{\circ}$ and extending to high latitudes is evident. This is caused by the combined emissions from CR nuclei and electrons interacting with the relatively nearby gas ( $\sim 100-500 \mathrm{pc}$ ) and the pile-up of the IC emissions from nearby and farther along the line-of-sight. The gas-related emissions are also higher because the CR densities in the ISM from the localised injection in the arms are more intense, which illuminate the gas that is nearby to these regions differently to the $\mathrm{CR}$ densities produced for the SA0 model. For the 3D CR/gas model sceario (right) the propagated CR spectra are not the same as for the reference model, being generally steeper in the ISM, because the consistency condition of normalising to the CR data requires propagation parameters and input source spectra that differ from the 2D case. Both scenarios produce enhanced IC and gas-related emissions compared to the standard 2D case because of the combined effects of the CR nuclei and lepton densities for the 3D source model, and the ISM densities. For the 3D CR source/ISM density models the enhancement is a density-squared effect because the CR and ISM densities multiply when determining the interstellar emissivities for the various $\gamma$-ray production processes

Comparison of the residual maps with those derived from high-energy $\gamma$-ray analysis, such as those shown in Fig. 2 of [11] (reproduced in the top left panel of Fig. 2) that used the SA0$\mathrm{Std} / 2 \mathrm{Dgas}$ combination as its baseline IEM, show similar spatial excesses at a level $\gtrsim 30 \%$ that extend out of the plane for $-45^{\circ}<l<45^{\circ}$. These are somewhat higher but similar in spatial distribution to those shown in Fig. 1 where there is a peak near $l \sim 45^{\circ}$ corresponding to the spiral arm enhancements together with an extension up to $|b| \sim 45^{\circ}$ latitudes. The excess of emission around $l \sim-30^{\circ}$ for the 3D model that is not evident in the residuals from subtracting the 2D IEM (left) is an indication that the equal injection power 4-arm model is too simplified a description for the spatial distribution of the CR sources.

Figure 1 also has a central 'hole' about the GC caused by the lower CR energy density in 

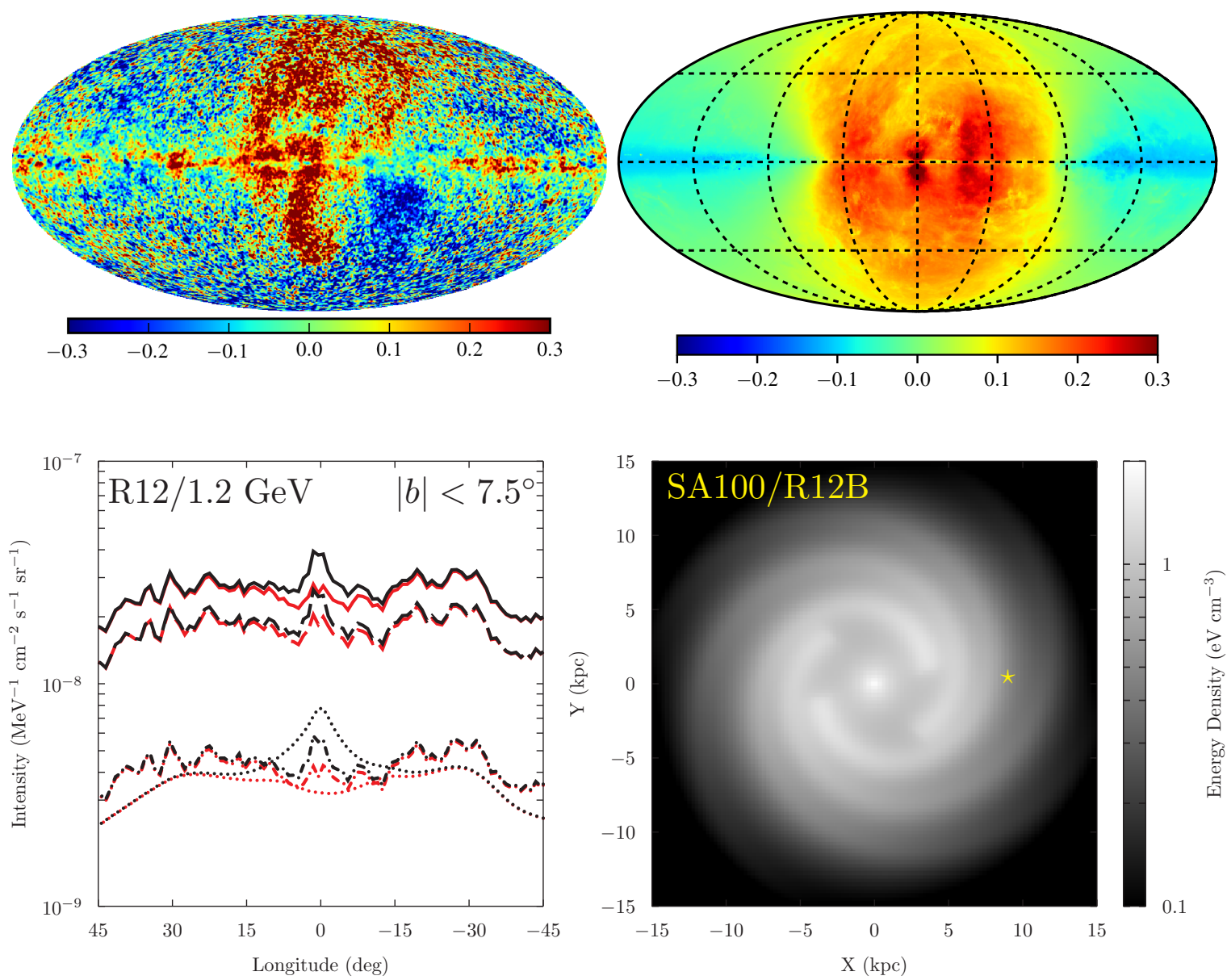

Figure 2: Top: Fractional residual map for $1-3.16 \mathrm{GeV}$ for the $\mathrm{SA} 0-\mathrm{Std} / 2 \mathrm{Dgas}$ model (left) derived from Fermi data by [11] and that for the SA100/R12B-R12/2Dgas model combination (right). Bottom: Longitude profile for the intensity at $1.2 \mathrm{GeV}$ averaged over $-7.5^{\circ} \leq b \leq 7.5^{\circ}$ (left) for the SA100-R12/2Dgas (red lines) and SA100/R12B-R12/2Dgas combination (black lines), respectively. Line-styles: solid, total; longdashed, $\pi^{0}$-decay; dash-dot, Bremsstrahlung; dotted, IC. Right panel shows spatial distribution of integrated CR energy density at the Galactic plane for the SA100/R12B CR source density model. The yellow star shows the location of the Solar system.

that region, whereas the residuals shown in Fig. 2 of [11] are $\gtrsim 30-40 \%$. A resolution to obtain comparable residual excesses that in-fill the region about the GC for the SA100 models is to introduce an extra source density model that provides additional CR power there. An explanation for such an additional component is that it could be a bulge/bar-related population of CR accelerators that injects both nuclei and leptons, or possibly only leptons. The first possibility is examined by recalculating the interstellar emissions with an additional CR central source density distribution following the R12/F98 stellar bulge/bar but with CR injection abundances the same as the arms for the SA100 density model. The fractional residual and the corresponding CR energy density spatial distribution for this 'what-if' scenario for the R12 bulge (termed SA100/R12B-R12/2Dgas) is shown for a $\gamma$-ray energy $1.2 \mathrm{GeV}$ in Fig. 2 (top right), where the injected CR power for the $\mathrm{R} 12$ bulge is $\sim 1 / 25$ of that from the $\mathrm{CR}$ arms. The residuals are $\sim 50 \%$ of the maximum of those 
from [11] so possibly a higher injected CR power is required. The corresponding longitude profile is shown in the bottom left panel of Fig. 2 where the density-squared effect of the combined CR lepton and ISRF densities about the GC produces a strong IC enhancement.

Fractional residual maps using the SA50 model together with the R12 or F98 bulge/bar CR source density, R12 or F98 ISRF, and 3D gas density model are shown in Fig. 3. The normalisation for the CR bulge/bar is the same as for Fig. 2. The full 3D calculations illustrate the sensitivity of the residual maps to the elements of Galactic structure related to the CR and ISM density distributions.

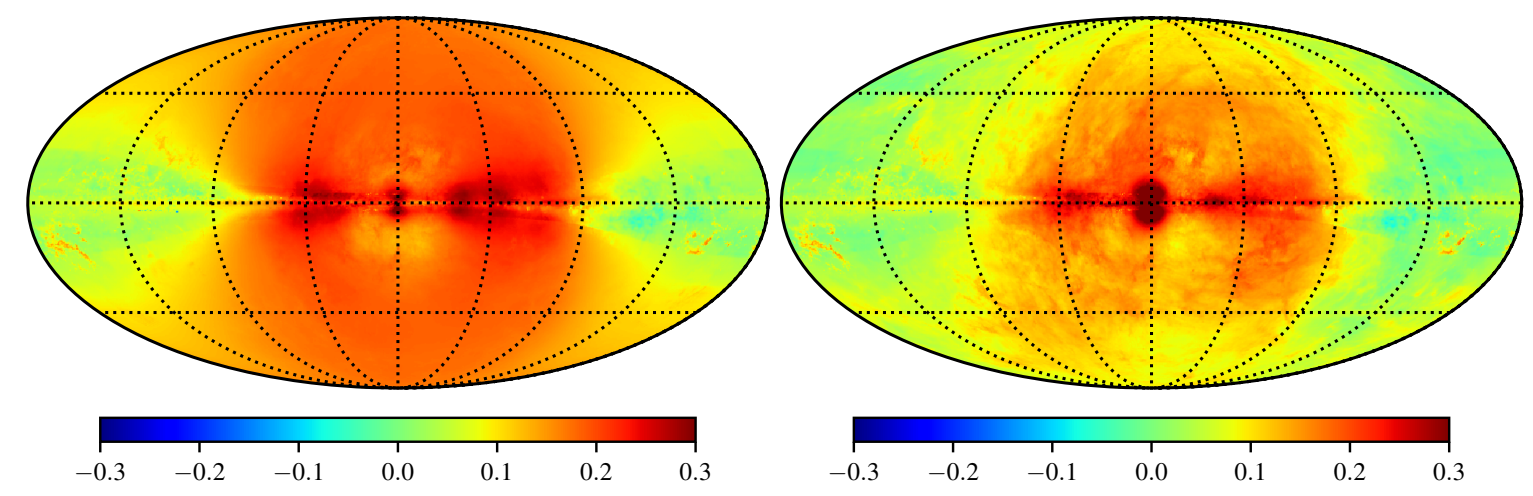

Figure 3: Fractional residual map at $1.2 \mathrm{GeV}$ for the SA50/R12B-R12/3Dgas (left) and SA50/F98BF98/3Dgas (right) CR source and ISM density models with respect to the SA0-Std/2Dgas reference case. The map is in Galactic coordinates with $l, b=0^{\circ}, 0^{\circ}$ at the centre. The longitude meridians and latitude parallels have $45^{\circ}$ spacing.

The modelling shown in Figs. 2 and 3 provide a framework for interpreting the results of [11]. For the inner $R \lesssim 3 \mathrm{kpc}$, the CRs injected by the bulge/bar in combination with the high ISRF spectral intensities over the region for the R12/F98 ISRF models result in a much higher IC intensity compared to the SA0-Std reference case. The multiplier effect enhances the IC by factors $\sim 2-3$, which is similar to the factor $\sim 4$ found by [11]. For $R \sim 3-8 \mathrm{kpc}$ the CR source density models with spiral arms the propagation smoothes the CRs injected by the arms into a quasi-axisymmetric ring (Fig. 2, bottom right). The CR energy densities over the region are $\sim 20-30 \%$ higher compared to the SA0 source model, which is similar to the scaling factors found also in the Fermi-LAT study.

This work has demonstrated the need for detailed modelling of the distribution of CR sources taking into account the 3D structure of the ISM. The residual structure in the calculated maps resemble features that have been previously interpreted as possible signs of new physics. However, further work is needed to optimise the models and more carefully tune them to the available data.

\section{Acknowledgments}

FRANKIE and GALPROP development is partially funded via NASA grant NNX17AB48G. Some of the results in this paper have been derived using the HEALPix [18] package. 


\section{References}

[1] I. V. Moskalenko and A. W. Strong, Production and Propagation of Cosmic-Ray Positrons and Electrons, ApJ 493 (Jan., 1998) 694-707, [astro-ph / 9710124 ].

[2] A. W. Strong and I. V. Moskalenko, Propagation of Cosmic-Ray Nucleons in the Galaxy, ApJ 509 (Dec., 1998) 212-228, [astro-ph/9807150].

[3] A. W. Strong, I. V. Moskalenko, and O. Reimer, Diffuse Continuum Gamma Rays from the Galaxy, ApJ 537 (July, 2000) 763-784, [astro-ph/9811296].

[4] A. E. Vladimirov, and et al., GALPROP WebRun: An internet-based service for calculating galactic cosmic ray propagation and associated photon emissions, Computer Physics Communications 182 (May, 2011) 1156-1161, [arXiv:1008.3642].

[5] M. Ackermann, and et al., Fermi-LAT Observations of the Diffuse $\gamma$-Ray Emission: Implications for Cosmic Rays and the Interstellar Medium, ApJ 750 (May, 2012) 3, [arXiv : 1202 .4039].

[6] A. W. Strong and I. V. Moskalenko, SNR and fluctuations in the diffuse Galactic $\gamma$-ray continuum, in Gamma 2001: Gamma-Ray Astrophysics (S. Ritz, N. Gehrels, and C. R. Shrader, eds.), vol. 587 of American Institute of Physics Conference Series, pp. 533-537, Oct., 2001.

[7] A. W. Strong and I. V. Moskalenko, A 3D time-dependent model for Galactic cosmic rays and gamma rays, International Cosmic Ray Conference 5 (Aug., 2001) 1964-1967, [astro-ph / 0106505 ].

[8] P. M. W. Kalberla, and et al., The Leiden/Argentine/Bonn (LAB) Survey of Galactic HI. Final data release of the combined LDS and IAR surveys with improved stray-radiation corrections, A\&A 440 (Sept., 2005) 775-782, [astro-ph/0504140].

[9] T. M. Dame, D. Hartmann, and P. Thaddeus, The Milky Way in Molecular Clouds: A New Complete CO Survey, ApJ 547 (Feb., 2001) 792-813, [astro-ph/ 0009217 ].

[10] T. A. Porter, G. Jóhannesson, and I. V. Moskalenko, High-energy Gamma Rays from the Milky Way: Three-dimensional Spatial Models for the Cosmic-Ray and Radiation Field Densities in the Interstellar Medium, ApJ 846 (Sept., 2017) 67, [arXiv: 1708.0081 ].

[11] M. Ajello, and et al., Fermi-LAT Observations of High-Energy Gamma-Ray Emission toward the Galactic Center, ApJ 819 (Mar., 2016) 44.

[12] G. Jóhannesson, and et al., Bayesian Analysis of Cosmic Ray Propagation: Evidence against Homogeneous Diffusion, ApJ 824 (June, 2016) 16, [arXiv: 1602.0224 ].

[13] M. Aguilar, and et al., Precision Measurement of the Proton Flux in Primary Cosmic Rays from Rigidity 1 GV to 1.8 TV with the Alpha Magnetic Spectrometer on the International Space Station, Physical Review Letters 114 (May, 2015) 171103.

[14] M. Aguilar, and et al., Electron and Positron Fluxes in Primary Cosmic Rays Measured with the Alpha Magnetic Spectrometer on the International Space Station, Physical Review Letters 113 (Sept., 2014) 121102.

[15] I. Yusifov and I. Küçük, Revisiting the radial distribution of pulsars in the Galaxy, A\&A 422 (Aug., 2004) 545-553, [astro-ph/0405559].

[16] F. J. Kerr and D. Lynden-Bell, Review of galactic constants, MNRAS 221 (Aug., 1986) 1023-1038.

[17] I. V. Moskalenko and A. W. Strong, Anisotropic Inverse Compton Scattering in the Galaxy, ApJ 528 (Jan., 2000) 357-367, [astro-ph/9811284].

[18] K. M. Górski, and et al., HEALPix: A Framework for High-Resolution Discretization and Fast Analysis of Data Distributed on the Sphere, ApJ 622 (Apr., 2005) 759-771, [a stro-ph/ 0409513 ]. 